\title{
The influence of interdependent self-construal on procedural fairness effects
}

\author{
Joel Brockner, ${ }^{\mathrm{a}, *, 1}$, David De Cremer ${ }^{\mathrm{b}, *, 1}$, Kees van den Bos ${ }^{\mathrm{c}}$, Ya-Ru Chen ${ }^{\mathrm{d}}$ \\ ${ }^{a}$ Columbia Business School, Columbia University, 715 Uris Hall, New York, NY, 10027, USA \\ ${ }^{\mathrm{b}}$ Department of Economic and Social Psychology, Tilburg University, P.O. Box 90153, 5000 LE Tilburg, The Netherlands \\ ${ }^{\mathrm{c}}$ Utrecht University, The Netherlands \\ ${ }^{\mathrm{d}}$ Rutgers University, USA \\ Received 6 February 2003 \\ Available online 15 December 2004
}

\begin{abstract}
Various theories have been shown to account for the effects of procedural fairness on people's attitudes and behaviors. We propose that a logical next step for organizational justice researchers is to delineate not whether, but rather when certain explanations are likely to account for people's reactions to procedural fairness information. Accordingly, the present research tested the hypothesis that social psychological explanations would be particularly applicable to people high in interdependent self-construal. As predicted, the results of three studies showed that interdependent self-construal (ISC) moderated the relationship between procedural fairness and a variety of dependent variables (cooperation, positive affect, and desire for future interaction with the other party). In different types of interpersonal encounters (social dilemmas, reward allocations, and negotiations), procedural fairness had more of an influence on participants' reactions among those high rather than low in ISC. Theoretical implications are discussed.
\end{abstract}

(C) 2004 Elsevier Inc. All rights reserved.

\section{Introduction}

Perceptions of fairness play an important role within work dyads and work teams, and as a consequence have received considerable attention from organizational and management scholars (Folger \& Cropanzano, 1998; Greenberg \& Cropanzano, 2001). Since the pioneering work of Thibaut and Walker (1975), it has been shown consistently that justice judgments are a function of not only how people perceive the distribution of outcomes

\footnotetext{
We are grateful to David Harrison and three anonymous reviewers for their constructive comments on an earlier version of the manuscript. The write-up of the present paper was supported by a grant from the Netherlands Organization for Scientific Research (NWO, Grant No. 016.005.019) awarded to David DeCremer.

* Corresponding authors. Fax: +31 134662067 (D. De Cremer).

E-mail addresses: jb54@columbia.edu (J. Brockner), D.deCremer@ uvt.nl (D. De Cremer)

${ }^{1}$ The first two authors contributed equally to this research; their order of authorship is alphabetical.
}

(i.e., distributive fairness) but also of how they perceive the procedures underlying the distribution of outcomes (i.e., procedural fairness; Brockner \& Wiesenfeld, 1996; Folger, 1986; Lind \& Tyler, 1988). Moreover, many studies have demonstrated that procedural fairness influences a variety of important work attitudes and behaviors, such as support and evaluations of authorities (Van den Bos, Wilke, \& Lind, 1998), compliance with authorities (Lind \& Tyler, 1988), organizational commitment (McFarlin \& Sweeney, 1992), organizational citizenship behavior (Moorman, 1991), and employee theft (Greenberg, 1990).

In addition to showing pervasive effects of procedural fairness on employees' attitudes and behaviors, researchers have offered a variety of explanations of these fair process effects. Initial theorizing accounted for people's desire for fair procedures from an instrumental perspective (Thibaut \& Walker, 1975). According to this viewpoint, fair procedures are preferred because they are expected to lead to more favorable tangible outcomes. 
Subsequent to the instrumental explanation, a number of social psychological models have been offered that focus on people's interactions and relationships with one another. Whereas the various social psychological explanations differ, one theme common to all of them is that people may be affected by procedural fairness information for reasons other than their implications for people's economic well-being (Folger, 1998). One such social psychological explanation is provided by the groupvalue model (Lind \& Tyler, 1988). According to this framework, people care about procedural fairness because it communicates information relevant to their sense of self and identity in their relationships with others. This model posits that the positive effects of voice arise because giving people the opportunity to express their opinion indicates that they are respected and valued by the party enacting the procedure. Such information, in turn, enhances people's sense of esteem and identity (e.g. Koper, van Knippenberg, Bouhuijs, Vermunt, \& Wilke, 1993).

Another social psychological viewpoint that has recently gained influence is the notion that fairness is a basic human value that people want to see affirmed in social encounters (Folger, 1998, 2001). This model suggests that people are concerned with procedural fairness not simply as a means towards better economic outcomes, nor as a means towards understanding their relationship with others and its resulting effects on one's sense of self and identity, but also as an end in its own right. Thus, because people hold ethical values (e.g., fairness) to be near and dear, they are very attentive to the issue of whether ethical values have been affirmed in the course of people's encounters with one another. This explanation can not only account for the fact that people directly involved in an exchange will be influenced by their perceptions of procedural fairness, but also that the attitudes and behaviors of people in the role of third party observers may be influenced by their procedural fairness perceptions (Folger, 2001; Lind, Kray, \& Thompson, 1998).

To date, theory and research have lent support to these and other explanations (e.g., Van den Bos \& Lind, 2002) of fair process effects (for a recent overview of explanations, see Van den Bos, in press). Therefore, it is clear that both the instrumental and the social psychological models help to explain people's reactions to their perceptions of procedural fairness. Accordingly, an important next step in the development of justice theory is to delineate the conditions under which certain explanations are likely to be especially influential. Put differently, rather than trying to determine which theory is "right" and which one is "wrong," justice researchers are likely to be better served by delineating when certain explanations are apt to be useful in accounting for people's reactions to procedural fairness information.
One way to do so is by examining the reactions of people who vary in the degree of importance that they assign to the factors central to a particular explanation. For example, the instrumental viewpoint should be particularly applicable to people who are highly concerned with material outcomes. The social psychological models should be particularly applicable to those who assign importance to their relationships with others for purposes of self-esteem and/or self-identity, as well as to those who strongly believe that ethical or moral values should be affirmed in social encounters. The present research focuses attention on the social psychological explanations by examining the influence of the dispositional variable known as interdependent self-construal on procedural fairness effects.

\section{Why focus on the moderating role of dispositional factors?}

Previous studies have shown that procedural fairness effects are particularly prominent under circumstances in which people attach greater psychological significance to their relationship with the other party (e.g., Tyler \& Degoey, 1995). More precisely, the more that people identified with the other party the more likely they were to be influenced by the other party's level of procedural fairness. It is worth noting, however, that the moderating influence tested for in previous research predominantly consisted of contextual or situational factors. In contrast, the present series of studies explores the moderating influence of a theoretically derived dispositional variable.

The social psychological explanations imply that the influence of procedural fairness on people's attitudes and behaviors should be more pronounced among those individuals who: (1) assign greater importance to their relationships with others for self-related reasons, and/or (2) more strongly believe that social interactions and relationships should affirm basic moral values. Moreover, these two factors may be related to one another. That is, those who assign importance to their relationships with others are in a potentially vulnerable position, in that they risk being exploited by the very parties towards whom they feel psychologically interdependent. This risk may be minimized to the extent that the other parties show that they can be trusted to act in ways that affirm basic moral values. One way in which to affirm basic moral values, in turn, is by being procedurally fair (Chen, Chen, \& Xin, 2004). Accordingly, for social psychological (rather than instrumental) reasons, ISC was expected to have an influence on the magnitude of procedural fairness effects. More specifically, the present studies tested the hypothesis that procedural fairness effects will be more strongly pronounced among those high rather than low in ISC.

The concept of ISC originally gained prominence in cross-cultural theory and research (Markus \& Kitayama, 1991). People high in ISC see themselves as more 
closely connected to others, especially ingroup members, relative to their counterparts who are low in ISC. One possible correlate of seeing oneself as closely connected to others is the belief that relationships with others should be respectful and affirm moral values. Although ISC is more predominant in collectivistic than individualistic cultures, more recent research has shown meaningful variation in people's collectivism-related beliefs (e.g., ISC) even within cultures (Chen, Brockner, \& Chen, 2002; Vandello \& Cohen, 1999). In other words, whereas culture is a broad and general concept that focuses on whether the norms society lives by are more versus less collectivistic (e.g., Hofstede, 1980), ISC reflects an individual-level construct referring to how much people define and evaluate themselves based on their relationships with significant others.

It is also worth noting that ISC is merely one dimension residing within the broader family of collectivism-related beliefs. In their exhaustive review of the individualism-collectivism literature, Oyserman, Coon, and Kemmelmeier (2002) identified eight different ways in which collectivism has been conceptualized. ${ }^{2}$ We chose to examine ISC because the essence of the construct pertains to factors central to several established social psychological explanations of procedural fairness effects (e.g., Folger, 1998; Lind \& Tyler, 1988).

\section{Study 1}

In Study 1 we examined the moderating influence of ISC on the element of procedural fairness that has received the most attention in the justice literature: voice. Voice refers to the opportunity people have to provide input into decision-making processes. Many studies have demonstrated that the extent to which people are allowed voice is a key determinant of procedural fairness (Lind \& Tyler, 1988; Thibaut \& Walker, 1975). Therefore, we will operationalize procedural fairness in Study 1 (and Study 2 to follow) by manipulating voice versus no-voice conditions. Individual differences in self-construal are assessed as an additional independent variable.

The dependent variable in Study 1 consisted of contribution behavior in the context of a social dilemma. Social dilemmas are defined as situations in which personal and collective interests are at odds with one another (Komorita \& Parks, 1994). Kramer (1991) has noted that social dilemmas occur frequently in work

\footnotetext{
${ }^{2}$ ISC is similar to the dimension that Oyserman et al. (2002) refer to as "Related," which they describe as the extent to which people consider close others an integral part of the self. Interested readers should refer to Oyserman et al. for a description of the other seven collectivism-related beliefs and hence, how they differ from ISC.
}

organizations (see also De Cremer \& Van Knippenberg, 2002). In social dilemma situations, people are highly interdependent on one another as their decisions influence both their own and others' outcomes.

A public goods dilemma was utilized in Study 1. Participants were asked to contribute towards a public good or service, which became available once a certain amount of money or points was invested. The dominant option in this type of social dilemma is to contribute as little as possible to the collective welfare because once the public good is provided no one can be excluded from using it. However, if all people behave this way then their collective outcomes will be worse than if all of them decided to contribute (Dawes, 1980). An interaction effect between voice and ISC was predicted. More specifically, the tendency for voice to be positively related to contribution behavior was expected to be more pronounced among those high rather than low in ISC.

\section{Method}

\section{Participants}

Seventy-nine students (42 men and 37 women) at Maastricht University in The Netherlands participated voluntarily and received course credit for doing so.

\section{Experimental procedure}

Participants were told that the study was an investigation about how organizational groups make decisions. Upon arrival at the room in which the study was conducted, participants were assigned into groups of five or six people. They were explicitly told not to communicate with one another. The groups were divided in such a way that it was salient to all participants that they were part of an actual group. Participants were seated at individual desks on which answer booklets were placed, and the anonymity and confidentiality of responses were emphasized. In the first part of the study, participants were asked to complete Singelis' (1994) 12-item measure of ISC. Sample items included, "I often have the feeling that my relationships with others are more important then my own accomplishments," and "I will sacrifice my self-interest for the benefit of the group I am in." Responses could range from "strongly disagree" (1) to "strongly agree" (7). Participants' responses were averaged into an index. Cronbach's $\alpha$ was .72, similar to the level observed in previous research (Oyserman et al., 2002; Singelis, 1994). As expected (given random assignment to the voice and no voice conditions), participants in the two conditions did not differ in their mean level of ISC, $t(74)=.82$, ns. Subsequently, participants were informed that their group resembled an organizational board consisting of six persons. They were then told that the task of this board was to make important decisions for the welfare of the organization (e.g., hiring people, regulating conflicts within the organization, etc.). 
They were told that the board needed a director to supervise and monitor several tasks and therefore one of them would be appointed to be the board leader. All information was communicated in a booklet given to participants at the beginning of the experiment. The appointment of the leader was made clear by a message that said that one of the group member's booklets included an extra page in which his or her task as board leader was explained. (In reality, however, none of the participants received such an extra page.) Participants were next told that their group would have to do several tasks. The first task would be to decide whom to hire as a new employee in their organization. It was made clear that in a later phase of the experiment they would receive several application forms on which to base the hiring decision.

Before starting the tasks, participants were required to answer a few questions about their perception of hiring procedures in organizations, and the experimenter then collected these answers. Thereafter, participants received an extra booklet, which contained information about the decision-making procedure. In the voice condition, participants were individually informed that the board leader had decided to ask his or her opinion regarding the hiring decision. Participants in the no-voice condition were individually informed that the leader had decided not to ask his or her opinion with respect to the hiring decision. To assess whether the procedure manipulation evoked differences in perceived procedural fairness, participants were asked to indicate on a sevenpoint scale how fair they considered the decision procedure of the board leader to be $(1=$ very unfair, $7=$ very fair).

Participants were then informed that their group entered a new fiscal quarter and therefore all board members would receive some organizational resources from a common pool. The resource that each board member received was 300 points (each point was worth 100 Dutch Guilders or approximately 50 US dollars). Participants were told that an investment plan would be introduced that would enable the organization to gain additional resources. According to this investment plan, each board member could invest or contribute some or all of the resources received into an investment plan. It was, however, important to realize that in a latter phase of the study, the leader would be able to monitor and regulate the distribution of the resources.

Participants were told that if the total sum of investments equaled or exceeded 900 points (the provision point was adjusted downward to 750 points if it were a five-person group), the total sum would be doubled and the leader would distribute that sum among all board members. Thus, the leader was responsible for the distribution of the outcomes and all board members would receive some part of the investment bonus, regardless of their contribution. As such, the leader would distribute the total amount contributed, but participants did not know exactly how the leader would distribute the amount amongst the different group members. However, if the total sum invested was less than 900 points (or 750 points, in a five person group), then all investments would be lost. Thus, each board member could lose his or her investment if the group as a whole did not contribute enough. As such, it was made clear that all group members were interdependent on one another. All participants were then asked how much they were willing to invest (ranging from 0 to 300 points). When the participants had answered this question, the study was finished and they were thoroughly debriefed and thanked.

\section{Results and discussion}

Correlational analyses showed that both ISC and voice were positively related to investment behavior (both $p$ values $<.001$ ), and that voice was unrelated to ISC.

\section{Manipulation check}

A one-way ANOVA on participants' perceptions of procedural fairness yielded a significant effect, $F(1,74)=117.60, p<.001, \eta^{2}=.61$. Inspection of the means indicated that, as expected, participants in the voice condition judged the procedure to be fairer $(M=5.58$, $S D=0.91 ; 95 \%$ confidence interval: $5.26-5.89)$ than participants in the no-voice condition $(M=3.13, S D=1.04$; 95\% confidence interval: $2.81-3.45$ ).

\section{Contribution behavior}

We performed a hierarchical regression analysis on participants' investments to test for the predicted interaction effect between voice and the centered ISC variable. The main effects of voice and ISC were entered simultaneously on the first step, and the interaction of the two was added on the second step. Of greatest importance, and as can be seen in Table 1, the predicted interaction effect was significant $(p<.05)$. To illustrate the nature of the interaction (cf. Aiken \& West, 1991), we computed the relationship between voice and contribution behavior at a high level of ISC (one SD above the

Table 1

Results of hierarchical regression analysis of contribution behavior as a function of ISC and voice (Study 1)

\begin{tabular}{lll}
\hline Terms & Model 1 & Model 2 \\
\hline ISC & $.36(.10)^{* *}$ & $.42(.32)$ \\
Voice & $.33(.10)^{* *}$ & $.33(.09)^{* *}$ \\
ISC $\times$ Voice & & $.82(.32)^{*}$ \\
$R^{2}$ & .26 & .32 \\
Overall $F$ & $13.37^{* *}$ & $11.75^{* *}$ \\
\hline
\end{tabular}

Note. Numbers in parentheses are standard errors of estimate. ${ }^{*} p<.05$.

${ }^{* *} p<.001$. 


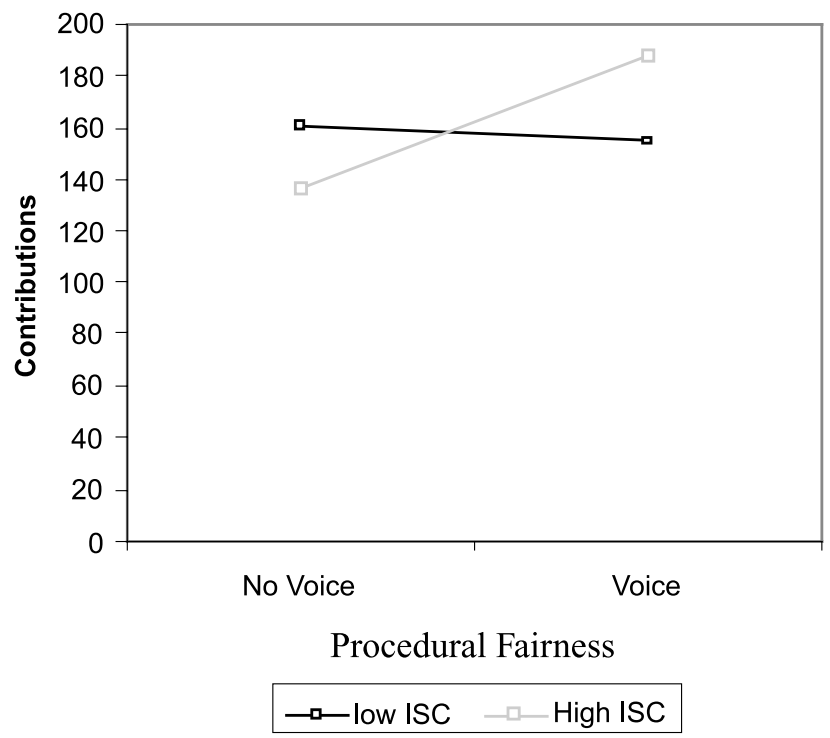

Fig. 1. The relationship between procedural fairness and contribution behavior as a function of ISC (Study 1).

mean) and at a low level of ISC (one SD below the mean). As can be seen in Fig. 1, the interaction took its predicted form. Voice was more positively related to investment behavior among those high rather than low in ISC. Simple slope analyses further revealed that among those high in ISC, voice was significantly related to contribution behavior, $\beta=.59, p<.005$, whereas among those low in ISC, the presence or absence of voice was not significantly related to contribution behavior, $\beta=-.08, p=.53$.

\section{Study 2}

Study 2 was designed to test for the generality of the interactive relationship between ISC and voice, by examining it in the context of a different type of interpersonal encounter (reward allocation), and by employing a different type of dependent variable (positive affect). Most justice studies of emotional reactions have examined negative affect, probably because injustice is believed to elicit feelings of anger and resentment (Folger \& Cropanzano, 1998; Mikula, Scherer, \& Athenstaedt, 1998). Mikula et al. (1998) suggest, however, that a wider range of emotions differing in valence also may be influenced by procedural fairness. Therefore, given the relative paucity of studies on how procedural fairness may influence people's positive emotional reactions (for a recent exception, see Van den Bos \& Spruijt, 2002), and given the pervasive influence of positive affect on employees' attitudes and behaviors (e.g., Isen \& Baron, 1991), positive affect served as the dependent variable in Study 1 . We expected to find a significant interaction between voice and ISC, such that the tendency for participants to respond with more positive affect when voice is high rather than low will be more pronounced among those high rather than low in ISC.

\section{Method \\ Participants}

Eighty-four students (16 men and 68 women) at the Free University in Amsterdam participated in the experiment and were paid for their participation.

\section{Experimental procedure}

Participants were invited to the laboratory to take part in a study on how people perform tasks. On arrival at the laboratory, participants were led to separate cubicles, each of which contained a computer with a monitor and a keyboard. Next to the monitor, participants found pieces of paper and a pencil. Participants were told that the computers were connected to one another and that the experimenter could communicate with them by means of the computer network. The computers were used to present the stimulus information and to collect data on the dependent variables and the manipulation checks. Participants took part in the experiment and answered the questions that constituted the dependent variables and the manipulation checks after participating in another, unrelated study. The studies lasted a total of approximately $105 \mathrm{~min}$, and participants were paid 20 Dutch guilders for their participation (1 Dutch guilder equaled approximately $\$ 0.50$ US at the time of the study).

After they had participated in the other study participants were informed that they would now take part in two additional studies that also were unrelated to each other. In the "first study" participants were asked to complete the Singelis (1994) measure of ISC used in Study 1. Responses ranged from strongly disagree (1) to strongly agree (7). Participants' responses to the measure of ISC were averaged into an index. Coefficient $\alpha$ was .69. Participants in the voice relative to the no voice conditions did not differ significantly in their level of ISC, $t(82)=.25, n s$, a finding that was expected in light of the fact that participants had been randomly assigned to the voice and no voice conditions.

After this, the "second study" was started. In the first part of the instructions, participants were informed that they would be taking part in the study with another person, referred to as Other. The experimental procedure was then outlined to the participants. After the experimental tasks were explained, participants were to practice the tasks for $2 \mathrm{~min}$, after which time they were to work on the tasks for $10 \mathrm{~min}$. Furthermore, participants were informed that after the study was completed a lottery would be held including all participants. The winner of this lottery would receive 100 Dutch guilders. (In fact, after all participants had completed the experiment, the 100 Dutch guilders were given to one participant, chosen 
at random.) Participants were told that a total of 200 lottery tickets would be divided among all participants. Furthermore, participants were told that after the work round the experimenter would divide some lottery tickets between them and Other. Seven practice questions were posed to ensure comprehension of the lottery. If participants gave a wrong answer to a question, the correct answer was disclosed and the main characteristics of the lottery were repeated.

The task was then explained to the participants. Figures were to be presented on the upper right part of the computer screen. Each figure consisted of 36 squares, and each square showed one of eight distinct patterns. On the upper left side of the computer screen one of the eight patterns would be presented, and participants had to count the number of squares with this pattern in the figure on the right side of the screen. When participants had indicated the correct number of patterns in the figure on the right side of the screen, another figure and another pattern would be presented on the screen. In both the practice round and the work round, the number of tasks that the participant had completed (i.e., the number of figures that the participant had counted) in the current round was presented on the lower right side of the screen. On the lower left side of the screen the time remaining in the present round was shown.

The practice round then began, after which the work round began. After the work round had ended, participants were told how many tasks they had completed in the work round, and -in order to make it more likely that participants compared themselves to Other-it was communicated to the participant that Other had completed an equivalent number of tasks. To assess whether participants thought of Other as a person who was comparable in the amount of inputs he or she provided, they were asked to what extent Other had performed well in the work round relative to the performance of the participant $(1=$ much worse, $4=$ equally, $7=$ much better), and to what extent Other was good in performing the tasks in the work round relative to the participant self $(1=$ much worse, $4=$ equally, $7=$ much better $)$. Participants were then asked to think for one minute about the percentage of lottery tickets that they should receive relative to Other.

After this, procedural fairness was manipulated. In the voice condition, the experimenter asked participants, by means of the computer network, to type in their opinion about the percentage of tickets that they should receive relative to Other. Participants in the no voice condition (which was explicit, see Van den Bos, 1999) were informed that they would not be asked to type their opinion about the percentage of tickets that they should receive relative to Other.

Participants were subsequently asked questions pertaining to the dependent variable and manipulation checks. All ratings were made on 7-point scales. Positive affect was assessed by asking participants to what extent they were in a positive mood $(1=$ very weak, $7=$ very strong), to what extent they felt proud $(1=$ very weak, $7=$ very strong), and to what extent they felt good about themselves $(1=$ very weak, $7=$ very strong). These dependent variables were strongly correlated and were averaged to form a scale of positive affect (Cronbach's $\alpha=.88$ ). To assess whether the manipulation of procedure evoked differences in perceived fairness, participants were asked how fair $(1=$ very unfair, $7=$ very fair $)$, just $(1=$ very injust, $7=$ very just $)$, and justified $(1=$ very unjustified, $7=$ very justified) they considered the way in which they had been treated. These three items were strongly correlated and were averaged to form a procedural fairness scale (Cronbach's $\alpha=.95$ ).

To check whether the voice manipulation had been perceived as intended, participants were asked to what extent they agreed with the statement that they had been given an opportunity to voice their opinion about the number of tickets they should receive relative to other, and to what extent they agreed with the statement that they had not been given an opportunity to voice their opinion about the number of tickets they should receive relative to other $(1=$ strongly disagree, $7=$ strongly agree $)$.

\section{Results}

Correlational analyses showed that the bivariate relationships between voice, ISC, and positive affect were not significant.

\section{Manipulation checks}

A one-way multivariate analysis of variance (MANOVA) on the two manipulation checks of voice (the voice check and the no-voice check) yielded highly significant effects at both the multivariate and the univariate levels: multivariate $F(2,82)=129.63, p<.001$, $\eta^{2}=.76$; for the voice check, $F(1,82)=240.38, p<.001$, $\eta^{2}=.75 ;$ for the no-voice check, $F(1,82)=173.40$, $p<.001, \eta^{2}=.68$. Participants in the voice condition agreed more with the statement that they had received an opportunity to voice their opinion $(M=5.83$, $S D=1.38,95 \%$ confidence interval: $5.40-6.26)$ than participants in the no-voice condition $(M=1.57, S D=1.13$, 95\% confidence interval: $1.22-1.92$ ). Participants in the no-voice condition agreed more with the statement that they had not received an opportunity to voice their opinion $(M=6.24, S D=1.43,95 \%$ confidence interval: 5.79 6.68) than participants in the voice condition $(M=2.07$, $S D=1.47,95 \%$ confidence interval: $1.61-2.53$ ). It can be concluded that the voice manipulation was successfully operationalized.

\section{Perceived procedural fairness}

A one-way ANOVA on the procedural fairness scale yielded a significant effect, $F(1,82)=126.58, p<.001$, 
$\eta^{2}=$.61. Inspection of the means indicated that, as expected, participants in the voice condition judged the procedure to be fairer $(M=5.56, S D=0.74,95 \%$ confidence interval: 5.33-5.78) than participants in the novoice condition $(M=2.94, S D=1.32,95 \%$ confidence interval: $2.53-3.35)$.

\section{Comparability measures}

As expected, participants' comparability judgments yielded no significant effects at both the multivariate level and the univariate levels. Participants indicated that the other participant had performed equally well in the work round $(M=3.98, S D=0.35,95 \%$ confidence interval: $3.90-4.05)$, and was equally good in performing the tasks $(M=3.96, S D=0.45,95 \%$ confidence interval: 3.87-4.06). Thus, participants thought of the other person as a comparable person with respect to the tasks that were completed in the experiment.

\section{Percentage findings}

Participants who were allowed voice $(n=42)$ typed in their opinion about the percentage of tickets that they should receive relative to the other participant. Virtually all of them (40 out of 42) indicated that the lottery tickets should be divided equally between themselves and the other participant, a finding that was expected in light of the fact that participants believed that they had performed at the same level as the Other.

\section{Dependent variable}

We performed a hierarchical regression on the measure of positive affect, in which the main effects of voice (coded as a dummy variable) and the centered (Aiken \& West, 1991) ISC variable were entered on the first step, followed by the interaction between the two on the second step. Table 2 shows the results of this analysis. Most relevant for the current purposes is that the interaction effect was significant $(p<.001)$. To illustrate the nature of the interaction, based on the procedures recommended by Aiken and West (1991) we computed the relationship between voice and positive affect at a high level of ISC (one SD above the mean) and at a low level of ISC (one $\mathrm{SD}$ below the mean). As predicted, and as can be seen in Fig. 2, the positive relationship between voice and positive affect was more pronounced among those high rather

Table 2

Results of hierarchical regression analysis of positive affect ratings as a function of ISC and voice (study 2)

\begin{tabular}{lll}
\hline Terms & Model 1 & Model 2 \\
\hline ISC & $.01(.18)$ & $.00(.17)$ \\
Voice & $-.36(.24)$ & $.36(.22)$ \\
ISC $\times$ Voice & & $-1.29(.34)^{*}$ \\
$R^{2}$ & .03 & .18 \\
Overall $F$ & 1.10 & $5.76^{*}$ \\
\hline
\end{tabular}

Note. Numbers in parentheses are standard errors of estimate. ${ }^{*} p<.001$.

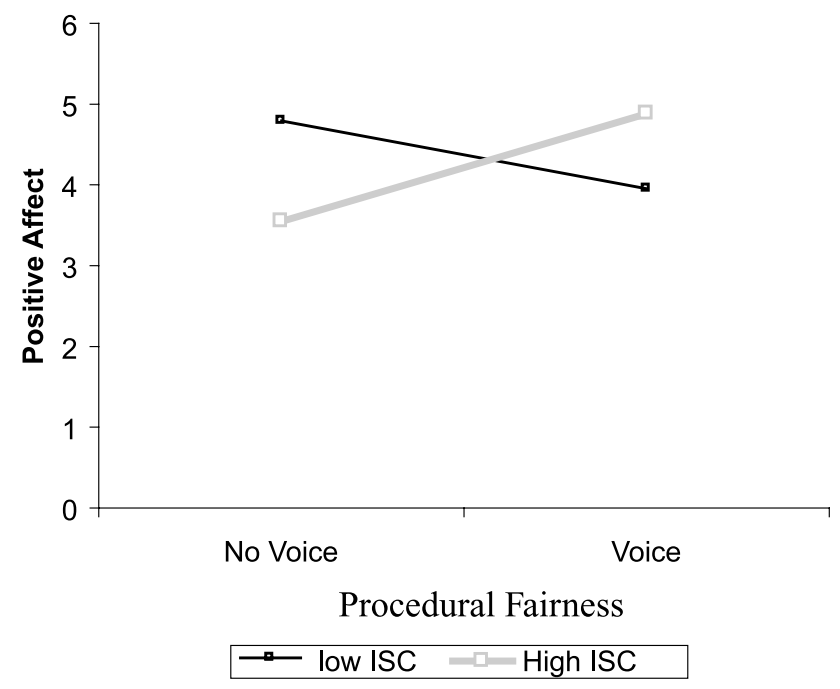

Fig. 2. The relationship between procedural fairness and positive affect as a function of ISC (Study 2).

than low in ISC. Simple slope analyses provide further insight into the nature of the interaction effect (Aiken \& West, 1991). Among those high in ISC, positive affect was significantly greater in the voice than in the no voice condition, $\beta=.61, p<.04$, whereas among those low in ISC the presence or absence of voice was not significantly related to positive affect, $\beta=-.35, p=.20$.

\section{Subsidiary analyses}

The psychometric properties of the ISC measure have been a matter of some controversy (e.g., Grace \& Cramer, 2003; Levine et al., 2003). Taken together, the results of Studies 1 and 2 provided some evidence that the Singelis (1994) measure was both reliable and valid. In both studies coefficient $\alpha$ s attained respectable levels, and ISC moderated the effects of voice in the theoretically expected direction. To further evaluate the uni-dimensionality of the ISC measure we conducted a confirmatory factor analysis (CFA), in which we evaluated the fit of a singlefactor model. In fact, the CFA did not provide evidence of a good fit, $\chi^{2}(54)=81.63, p<.01$; root means square error of approximation $=.08$; root mean square residual $=.17$; comparative fit index $=.74$. Post hoc inspection showed that the first three items appearing in Singelis' (1994) description of the measure did not load well. Upon removing these three items from the CFA, we found that the single-factor solution provided a much better fit, $\chi^{2}$ $(27)=34.91, \quad(p>.10)$; root means square error of approximation $=.06$; root mean square residual $=.17$; comparative fit index $=.90$. Furthermore, when we-ran the multiple regression analysis in which the first three items from the ISC had been deleted, the interaction effect between ISC and voice became even larger, with its $F$ ratio growing from 14.75-19.00. Given the unexpected effects associated with deleting the first three items from the ISC measure, we re-evaluated them in upcoming Study 3. 
Participants in Study 1 also completed the Rosenberg (1965) self-esteem measure, which was included in the same survey that contained the ISC. Self-esteem was significantly related to ISC, $r=-.36, p<.01$, albeit moderately so. We also conducted a regression analysis to determine whether self-esteem moderated the relationship between voice and positive affect. Importantly, the interaction between self-esteem and voice did not even approach significance $(p>.20)$, thereby providing some evidence of the discriminant validity of the measure of ISC.

\section{Discussion}

As predicted, the results of Study 2 showed that those participants high in ISC reacted more positively when voice was allowed than when voice was denied. No such difference was found for those low in ISC. Although the field of organizational justice has reported effects of fairness perceptions on people's affective reactions (e.g., Weiss, Suckow, \& Cropanzano, 1999), affective reactions are used relatively infrequently as dependent variables in the justice literature (Tyler \& Smith, 1998). The present findings show that it is important to assess positive affect not only because of its pervasive effects on people's work attitudes and behaviors (e.g., Isen \& Baron, 1991), but also because ISC moderated the effect of voice on people's level of positive affect.

The enhancing effect of voice on people's attitudes and behaviors is one of the most robust findings in the procedural justice literature (e.g., Lind \& Tyler, 1988; Thibaut \& Walker, 1975). Given the generally positive effects of voice that have been found in past research, it is noteworthy that giving voice to those low in ISC did not have any influence on their affective states in Study 2 (and on their contribution behavior in Study 1).

Furthermore, the findings provide insight into the important question of not whether, but rather when procedural fairness effects may be accounted for by certain social psychological explanations. That is, social psychological explanations of the positive effect of giving people voice seem to be particularly applicable to those relatively high in ISC and not to those low in ISC.

\section{Study 3}

Study 3 was designed to evaluate further the interactive relationship between voice and ISC. In particular, the generality of the results of Studies 1 and 2 was evaluated in four ways. First, Study 3 was based on a different conception (and operationalization) of procedural fairness. In Studies 1 and 2, procedural fairness consisted of voice. Whereas voice is one element or determinant of procedural fairness, it is by no means the only one (Leventhal, 1980). For example, people's perceptions of procedural fairness also depend upon the quality of the interpersonal treatment that they experience in their interactions with others. Indeed, the very core of certain social psychological explanations of fair process effects suggests that people's attitudes and behaviors should be influenced by the fairness of their interpersonal treatment (Bies, 1987), which both informs people about the quality of their relationships with the other party, and reflects the extent to which moral values have been affirmed in the course of their social encounters. Moreover, the social psychological explanations suggest that the effects of fairness of interpersonal treatment should be especially pronounced among those higher in ISC, who attach greater psychological significance to (i.e., define themselves based on) their relationships with others, and in so doing, may more strongly prefer that moral values are affirmed in their social encounters. Accordingly, the fairness of the interpersonal treatment that participants experienced served as the conceptualization of procedural fairness in Study 3 . We expected its influence on the dependent variable (described below) to be more pronounced among those high rather than low in ISC.

Second, the focal interaction between procedural fairness and ISC was examined in a different social context, relative to Studies 1 and 2. More specifically, participants in Study 3 took part in a negotiation exercise. Third, the dependent variable in Study 3 differed, relative to that assessed in the earlier studies. Specifically, participants rated their desire for future interaction with their negotiation partner, which may be considered to be a proxy for their judgments of the quality of their encounter with the other. Finally, Study 3 was conducted in a different cultural context (Japan), thereby enabling us to evaluate whether the expected interaction between procedural fairness and ISC may be found in an Asian (rather than European) context.

In summary, all participants in Study 3 took part in a negotiation with one other person. Independent variables consisted of ISC as well as the perceived fairness of the interpersonal treatment that participants received from their negotiation partner, and the dependent variable was participants' desire for future interaction with their negotiation partner. We predicted that the positive relationship between fairness of interpersonal treatment and desire for future interaction with the other party would be significantly stronger among those high rather than low in ISC. Indeed, strong evidence for our hypothesis would be revealed if, in spite of the many differences between Study 3 and its predecessors we were to find an interaction effect conceptually analogous to those found in the earlier studies.

\section{Method}

\section{Participants}

Participants were 118 senior undergraduate business majors at Shiga University in Japan. They participated 
in the negotiation study as part of requirements for a management course.

\section{Experimental procedure}

Upon arrival at the negotiating session, all participants were asked to fill out a pre-negotiation questionnaire that included the measure of ISC. After they completed this questionnaire, participants were randomly assigned to play the role of either the executive vice-president of an outdoor sports manufacturer (seller) or the executive vice-president of a company that provides protective fire-fighting clothing to fire departments (buyer) in a dyadic, mixed-motive negotiation. They were given $30 \mathrm{~min}$ to read their role materials and a maximum of $40 \mathrm{~min}$ to complete the negotiation.

Upon finishing the negotiation, all participants were given a post-negotiation questionnaire which measured their perceptions of the fairness of their partner's interpersonal treatment towards them, their desire for future interaction with the negotiation partner, and a control variable to be described below.

\section{Task overview}

The experimental negotiation task, called Z-Tec, was adapted from a negotiation simulation described by Bazerman and Neale (1992, p. 85). To assess the clarity and appropriateness of the simulation, we pre-tested it with 10 Japanese business school students at Shiga University, all of whom indicated that the description was clear and the negotiation context was not unusual.

All materials were administered in Japanese. In developing the Japanese versions of the materials, we employed the back-translation procedure (Brislin, 1980). That is, the materials were first translated from English to Japanese by a Japanese graduate student who was also fluent in English. The versions then were translated back to English by a graduate student from the United States who was also fluent in Japanese. The new English version was then compared with the original; any discrepancies arising through back translation process were then adjusted.

\section{Questionnaire measures}

ISC

The 12-item Singelis (1994) measure used in the previous two studies was also employed in this study. All responses were recorded on a seven-point rating scale with endpoints labeled "strongly disagree" (1) and "strongly agree" (7). Coefficient $\alpha$ was .73.

\section{Fairness of interpersonal treatment}

The extent to which participants felt that they were treated fairly by the other party was assessed with a three-item measure: (1) "In your opinion, how fairly did the other party treat you?", (2) "How much respect and dignity did the other party show to you during the negotiation?", and (3) "How often did the other party show attempts to understand your concerns and interests during the negotiation?". Responses on the seven-point rating scale could range from "not at all" (1) to "very much" (7). Coefficient $\alpha$ was .87.

\section{Desire for future interaction}

The desire to engage in future interaction with their negotiating partner was assessed with participants' responses to the following item: "How much do you want to continue any future business dealings with the same party?" Endpoints on the seven-point rating scale were "not at all" (1) and "very much" (7).

\section{Control variables}

Social exchange theory (e.g., Blau, 1964) and empirical research (e.g., Brockner, Chen, Mannix, Leung, \& Skarlicki, 2000) has shown that people's desire for ongoing interaction with one another depends upon the favorability of their prior encounters. Accordingly, we included a four-item measure of participants' perceived favorability of their negotiation outcome, e.g., "How satisfied are you with your individual profit level?" Responses, which could range from "not at all" (1) to "very much" (7), were averaged into an index. Coefficient $\alpha$ was. 73. We also controlled for the role that participants played during the negotiation (buyer or seller).

\section{Results}

Summary statistics and intercorrelations are shown in Table 3. Perhaps most noteworthy is that ISC was uncorrelated with the measures of procedural fairness, outcome favorability, and desire for future interaction, even though all of the measures were assessed with a common method (self-report).

The hypothesis was tested with the use of a hierarchical regression analysis. In the first step we entered the control variables of outcome favorability and role, and the main effects of the centered variables of fairness of interpersonal treatment and ISC. On the second step we added the interaction between treatment fairness and ISC. Of greatest importance, and as can be seen in Table 4, the interaction effect was significant $(p<.05)$.

Table 3

Means, standard deviations, and intercorrelations of Study 3 variables

\begin{tabular}{|c|c|c|c|c|c|c|c|}
\hline Variables & $M$ & $S D$ & 1 & 2 & 3 & 4 & 5 \\
\hline $\begin{array}{l}1 \text { Desire for future } \\
\text { interaction }\end{array}$ & 5.07 & 1.39 & - & & & & \\
\hline 2 Outcome favorability & 4.25 & 1.13 & $.32^{*}$ & - & & & \\
\hline 3 Procedural fairness & 4.69 & 0.85 & $.30^{*}$ & $.30^{*}$ & - & & \\
\hline 4 ISC & 4.39 & 0.67 & .06 & .08 & .07 & - & \\
\hline 5 Role & 0.50 & 0.50 & .04 & -.07 & -.01 & .09 & - \\
\hline
\end{tabular}


Table 4

Results of hierarchical regression analysis of desire for future interaction as a function of ISC and fairness of treatment (Study 3)

\begin{tabular}{lll}
\hline Terms & Model 1 & Model 2 \\
\hline Role & $-.18(.24)$ & $-.16(.24)$ \\
Outcome favorability & $.28(.11)^{*}$ & $.31(.11)^{* *}$ \\
ISC & $.25(.20)^{*}$ & $-2.81(1.16)^{*}$ \\
Fairness of treatment & $.31(.15)^{*}$ & $-1.69(.79)^{*}$ \\
ISC $\times$ Fairness of treatment & & $.55(.24)^{*}$ \\
$R^{2}$ & .15 & .20 \\
Overall $F$ & $5.14^{* * *}$ & $5.61^{* * *}$ \\
\hline
\end{tabular}

Note. Numbers in parentheses are standard errors of estimate.

${ }^{*} p<.05$.

** $p<.01$.

*** $p<.001$.

To illustrate the nature of the interaction effect we computed the relationship between fairness of interpersonal treatment and desire for future interaction at a high level of ISC (one SD above the mean) and at a low level of ISC (one SD below the mean; Aiken \& West, 1991). Fig. 3 shows that the interaction effect took its predicted form, in which treatment fairness and desire for future interaction were more positively related to one another when ISC was high rather than low. Furthermore, simple slope analyses showed that the positive relationship between fairness of treatment and desire for future interaction was significant when ISC was high $(\beta=1.57, p<.05)$, and that treatment fairness and desire for future interaction were unrelated when ISC was low $(\beta=0.70, n s) .^{3}$

\section{Subsidiary analyses}

As in Study 2, we conducted a confirmatory factor analysis on the measure of ISC, evaluating the fit of a single-factor solution. As in Study 2, the single-factor solution did not provide evidence of a good fit: $\chi^{2}$ $(55)=87.32, \quad p<.01$; root means square error of approximation $=.07$; root mean square residual $=.25$; comparative fit index $=.51$. Prompted by the CFA results in Study 2, we evaluated whether deleting the first three items from the Singelis (1994) measure had the effect of improving the fit of a single-factor solution. In fact, it did; $\chi^{2}(27)=29.54, p>.30$; root means square error of approximation $=.03$; root mean square residual $=.15$; comparative fit index $=.93$. Moreover, as in Study 2, when we re-ran the regression analysis delet-

\footnotetext{
${ }^{3}$ In light of research showing an interaction effect between procedural fairness and outcome favorability (Brockner \& Wiesenfeld, 1996), we conducted an additional step to the hierarchical regression, in which the interaction between fairness of interpersonal treatment and outcome favorability was added to the interaction term already entered in Table 4, Step 2. In fact, the interaction between fairness of interpersonal treatment and outcome favorability was not significant. Of perhaps greater importance, however, adding the interaction between fairness of interpersonal treatment and outcome favorability had no effect on the significance level of the interaction between fairness of interpersonal treatment and ISC, which remained significant at the .05 level.
}

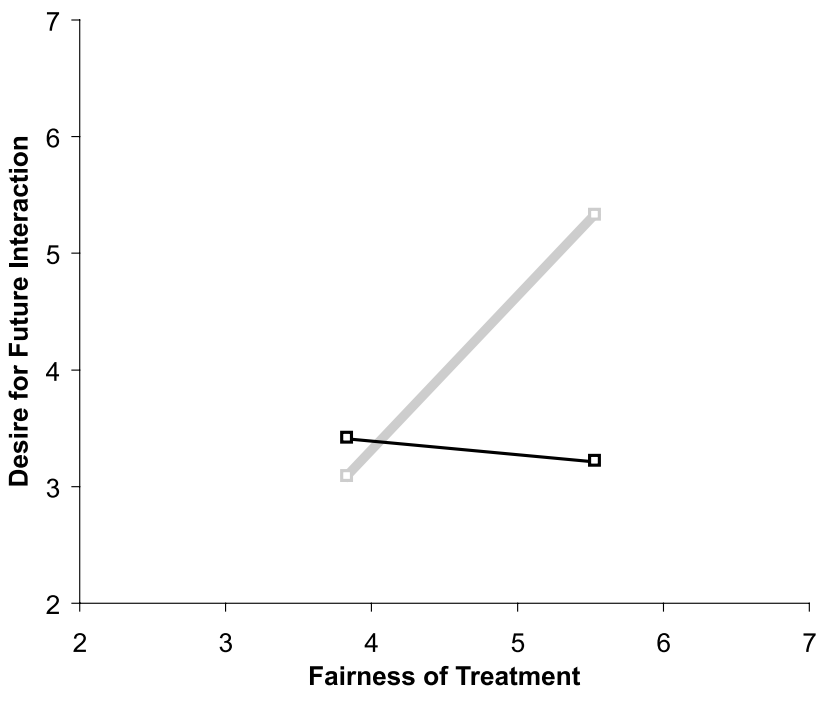

high ISC $\quad-\square-$ low ISC

Fig. 3. The relationship between fairness of treatment and desire for future interaction as a function of ISC (Study 3).

ing the first three items from the ISC measure, the interaction effect between ISC and Fairness of Treatment grew in magnitude (from an $F$ of 5.15 to an $F$ of 5.95).

\section{Discussion}

One of the shortcomings of Study 3 is that the dependent variable consisted of a single item measure. In response to this concern, we raise the following two points. First, the results of Study 3 are buttressed by the results of Studies 1 and 2, in which highly similar results emerged. In Study 1, the dependent variable was a behavioral measure of cooperation and in Study 2 the dependent variable was a multiple-item measure of positive affect with high internal consistency. The fact that similar results emerged across all three studies strongly suggests that the results of Study 3 are not an artifact of the dependent variable consisting of a single item measure. Second, the same item used in Study 3 is actually one of three items used to measure desire for future interaction with the other party in other research (Chen $\&$ Chen, 2004). The other two items in that study were: (a) "How much are you willing to introduce this negotiating person to your future company as a client?", and (b) "How much are you willing to negotiate (or partner) with the same negotiating person in future in-class negotiation simulations?" Importantly, all three items were highly interrelated, as indicated by the coefficient $\alpha$ of .93. Thus, the single item measure used in Study 3 is highly related to other face valid measures of desire for future interaction with the other party in a negotiation setting. Nevertheless, future research would be better served by the use of multi-item scales, or, more generally, other sorts of measures with proven construct validity. 


\section{General discussion}

Taken together, the findings show that perceptions of procedural fairness have very different effects on people high versus low in ISC. In all three studies, participants reacted strongly to procedural fairness information when they were high in ISC, but not when they were low in ISC. Brockner, Ackerman, and Fairchild (2001) suggested that one way to achieve conceptual progress in the organizational justice literature is by identifying the conditions under which procedural fairness factors are more versus less impactful. Accordingly, the present research joins a growing list of studies that have delineated pervasive moderators of the effects of procedural justice such as outcome favorability (e.g., Brockner \& Wiesenfeld, 1996) and uncertainty (e.g., Van den Bos \& Lind, 2002).

Further, the present findings also demonstrate the robustness of the moderating influence of ISC. First, we found the effect in three different types of interpersonal encounters, that is, social dilemmas, reward allocations, and negotiations. Second, the present studies used different elements of procedural fairness (voice and fairness of interpersonal treatment), and showed that ISC had a similar moderating influence on both elements. Indeed, Leventhal (1980) argued that multiple factors may influence procedural fairness and that research needs to focus on all of them to reveal a better understanding of the psychology of procedural justice. In a similar vein, Brockner et al. (2001) suggested that it is important to evaluate whether moderators of procedural fairness effects are limited to a single determinant of procedural fairness, or whether they apply to multiple determinants of procedural fairness. The present findings suggest that ISC as a moderator certainly is not limited to a single aspect of procedural fairness.

Third, the moderating effect of ISC on procedural fairness was also found on different dependent measures. Our studies demonstrated that variations in fairness of procedures influenced people's contribution behavior, positive affect, and desire for future interaction, particularly if they were high in ISC. The fact that converging results emerged on an assortment of dependent variables suggests that the findings have implications for a number of domains of organizational behavior. Finally, this interaction effect was demonstrated in two different cultures, the Netherlands (Europe) and Japan (Asia). In short, the similarities in findings that emerged in the face of all these noteworthy differences bodes well for the construct validity of: (1) the independent variables of procedural fairness and ISC, and (2) the influence of ISC on procedural fairness effects.

Importantly, the findings of all three studies speak to the question of when certain social psychological explanations may best account for procedural fairness effects. That is, the present research was designed not to exam- ine whether social psychological explanations of fair process effects are more appropriate than others (e.g., instrumental perspectives) that have gained traction in the organizational justice literature. Rather, the present research was designed to begin the important task of delineating the conditions under which or the people for whom social psychological explanations are appropriate. Indeed, the choice of ISC as a moderator variable was motivated by prior theory and research suggesting that people are affected by procedural fairness because it communicates information about how they are viewed by the other party, and because it says something about how much basic moral values are being affirmed in the course of the encounter. Hence, people who assign greater importance to these social psychological considerations (namely, those high in ISC) were expected to be more affected by the level of procedural fairness that they perceived.

The present studies also contribute to the organizational justice literature on the basis of the dependent variables that were assessed. Recently, a strong plea has been made to examine how perceptions of fairness influence affective reactions (Tyler \& Smith, 1998; Van den Bos \& Miedema, 2000; Weiss et al., 1999). Although justice is quite often thought of as an affective event, most social and organizational justice research has, indeed, neglected this response variable. This tendency is rather disappointing because emotions are a fundamental aspect of life in work organizations (Isen \& Baron, 1991), and therefore warrant careful study (e.g., Brief, 2001). The results of Study 2 showed that voice influenced participants' affective reactions, particularly among those high in ISC. Also, research on interpersonal relationships has shown that positive emotions are experienced more when belongingness needs are met (e.g. Baumeister \& Leary, 1995), an assumption that is in line with the present finding that among those relatively high in ISC the presence or absence of voice had more of an effect on their levels of positive affect.

Furthermore, the voice manipulation in Study 1 also influenced cooperative behavior in a social dilemma situation. Much of the research on procedural fairness in organizational settings has not assessed its impact on the important behavioral outcome of cooperation (see Smith, Carroll, \& Ashford, 1995; for the importance of cooperation in organizational settings). The present findings show that in organizational public goods dilemmas, perceived voice influenced contribution decisions, particularly among those high in ISC (cf. De Cremer \& Van Vugt, 2002).

Before closing we should mention some limitations of the present research, and thereby suggest some possible leads for future research. One of the shortcomings pertains to the conceptualization and assessment of ISC. Recently, several criticisms of the construct have been raised, stressing the fact that a sole focus on the social 
self in the definition of self-construal is too limited (e.g., Fiske, 2002). Furthermore, there is considerable debate concerning the assessment of ISC (Grace \& Cramer, 2003; Levine et al., 2003). The fact that CFAs yielded similar results in Studies 2 and 3, and the fact that the primary results became even stronger when the first three items from the Singelis (1994) measure had been deleted, suggests that further research is needed to clarify both the conceptualization and operationalization of ISC. For example, future research should clarify the distinctions between ISC and the broader family of collectivism-related beliefs documented by Oyserman et al. (2002).

Second, whereas the moderating effect of ISC on procedural fairness was highly consistent across a very diverse set of studies, some between-study inconsistencies in the findings warrant further attention. For example, ISC yielded a significant main effect in Study 1 only. Why might that have been the case? Because ISC reflects the importance people assign to their relationships, those high in ISC might strategically choose to behave in ways that foster more positive, enjoyable, and cooperative relationships. Note, that the dependent variables in the three studies may have differed in the extent to which they gave participants the opportunity to foster more positive, enjoyable, and cooperative relationships. The dependent variable in Study 2 consisted of an affective measure, whereas the dependent variable in Study 3 consisted of an attitudinal measure (desire for future interaction). In contrast, the dependent variable in Study 1 was a behavioral measure of cooperation. Perhaps participants in Study 1 felt that they might have been able to influence the emerging relationship with their fellow participants (to be more cooperative) if they were to behave cooperatively themselves. If so, then the tendency to behave cooperatively should be more likely to be exhibited by those high in ISC. Further research is needed to evaluate this speculation.

A third potential limitation is that the research settings may have afforded relatively low levels of contextual realism or meaning for the participants. For example, in Study 1, participants did not have expectations for future interactions, were a member of a fictitious company, and did not know the group leader. In addition, participants received a written note with respect to the voice procedure. Thus, the testing conditions in the present studies were not representative of the kinds of interactions that take place in contemporary organizations. Future field research is needed to examine the present predictions under more realistic (and engaging) conditions.

Fourth, the present studies merely looked at the moderating impact of interdependent self-construal but not at the moderating effect of independent self-construal (Markus \& Kitayama, 1991). Being high in independent self-construal refers to seeing "oneself as an individual whose behavior is organized and made meaningful primarily by reference to one's own internal repertoire of thoughts, feelings, and actions, rather than by reference to the thoughts, feelings, and actions of others" (Markus \& Kitayama, 1991, p. 226). Singelis (1994) has shown that interdependent and independent self-construals represent two distinct dimensions, rather than being endpoints on a single dimension. Whereas the present study shows that interdependent self-construal has a moderating influence on procedural fairness, future research needs to evaluate whether the same may be said for independent self-construal.

Fifth, we focused on two distinct social psychological explanations of procedural fairness effects, one emphasizing the implications of relational considerations for people's sense of self, and the other focused on people's desire to see basic moral values affirmed in social encounters. Whereas these two factors may be related to one another, they are conceptually distinct. Thus, future research is needed to delineate when one of these social psychological explanations is more or less likely to account for the effects of procedural fairness on employees' cognitions, emotions, and behavior.

In conclusion, the present studies make an important contribution to the organizational justice literature by showing a highly consistent influence of a theoretically derived individual difference variable, interdependent self-construal, on procedural fairness effects. In so doing, we also help to identify not whether, but rather when certain social psychological explanations may be particularly well-suited to account for the pervasive effects of procedural fairness information.

\section{References}

Aiken, L. S., \& West, S. G. (1991). Multiple regression: Testing and interpreting interactions. New York: Sage.

Baumeister, R. F., \& Leary, M. R. (1995). The need to belong: Desire for interpersonal attachments as a fundamental human motivation. Psychological Bulletin, 117, 497-529.

Bazerman, M. H., \& Neale, M. A. (1992). Negotiating rationally. New York: Free Press.

Bies, R. J. (1987). The predicament of injustice: The management of moral outrage. In L. L. Cummings \& B. M. Staw (Eds.), Research in organizational behavior (Vol. 9, pp. 289-319). Greenwich, CT: JAI Press.

Blau, P. (1964). Exchange and power in social life. New York: Wiley.

Brief, A. P. (2001). Organizational Behavior and the study of affect: Keep your eyes on the organization. Organizational Behavior and Human Decision Processes, 86, 131-139.

Brislin, R. W. (1980). Translation and content analysis of oral and written material. In H. C. Triandis \& J. W. Berry (Eds.), Handbook of cross-cultural psychology (Vol. 2, pp. 389-444). Boston: Allyn \& Bacon.

Brockner, J., Ackerman, G., \& Fairchild, G. (2001). When do elements of procedural fairness make a difference? A classification of moderating influences. In J. Greenberg \& R. Cropanzano (Eds.), Advances in organizational justice (pp. 179-212). Stanford, CA: Stanford University Press. 
Brockner, J., Chen, Y., Mannix, E., Leung, K., \& Skarlicki, D. (2000). Culture and procedural fairness: When the effects of what you do depend upon how you do it. Administrative Science Quarterly, 45, 138-159.

Brockner, J., \& Wiesenfeld, B. M. (1996). An integrative framework for explaining reactions to decisions: Interactive effects of outcomes and procedures. Psychological Bulletin, 120, 189-208.

Chen, Y., Brockner, J., \& Chen, X. (2002). Individual-collective primacy and ingroup favoritism: Enhancement and protection effects. Journal of Experimental Social Psychology, 38, 482-491.

Chen, Y., Chen, X. (2004). Reciprocating positive versus negative inequitable offers: Culture, relationships, and emotions. Manuscript under editorial review, Rutgers University.

Chen, C., Chen, Y., \& Xin, K. (2004). The downside of managerial guanxi practice in the People's Republic of China: A procedural justice perspective. Organization Science, 15, 200-209.

Dawes, R. M. (1980). Social dilemmas. Annual Review of Psychology, 31, 169-193.

De Cremer, D., \& Van Knippenberg, D. (2002). How do leaders promote cooperation? The effects of charisma and procedural fairness. Journal of Applied Psychology, 87, 858-866.

De Cremer, D., \& Van Vugt, M. (2002). Intergroup and intragroup aspects of leadership in social dilemmas: A relational model of cooperation. Journal of Experimental Social Psychology, 38, 126136.

Fiske, A. (2002). Using individualism and collectivism to compare cultures-A critique of the validity and measurements of the constructs: Comment on Oyserman et al. 2002. Psychological Bulletin, $128,78-88$.

Folger, R. (1986). Rethinking equity theory: A referent cognitions model. In H. W. Bierhoff, R. L. Cohen, \& J. Greenberg (Eds.), Justice in social relations (pp. 145-162). New York: Plenum Press.

Folger, R. (1998). Fairness as a moral virtue. In M. Schminke (Ed.), Managerial ethics: Moral management of people and processes (pp. 13-34). Mahwah, NJ: Erlbaum.

Folger, R. (2001). Fairness as deonance. In S. W. Gilliland, D. D. Steiner, \& D. P. Skarlicki (Eds.), Research in social issues in management (Vol. 1, pp. 3-33). New York: Information Age Publishers.

Folger, R., \& Cropanzano, R. (1998). Organizational justice and human resource management. Thousand Oaks, CA: Sage.

Grace, S. L., \& Cramer, K. L. (2003). The elusive nature of self-measurement: The self-construal versus the twenty statements test. Journal of Social Psychology, 143, 649-668.

Greenberg, J. (1990). Organizational justice: Yesterday, today, and tomorrow. Journal of Management, 16, 399-432.

Greenberg, J., \& Cropanzano, R. (2001). Advances in organizational justice. Stanford, CA: Stanford University Press.

Hofstede, G. (1980). Culture's consequences: International differences in work-related values. Beverly Hills, CA: Sage.

Isen, A. M., \& Baron, R. A. (1991). Positive affect as a factor in organizational behavior. In L. Cummings \& B. M. Staw (Eds.), Research in organizational behavior (Vol. 13, pp. 1-53). Greenwich, CT: JAI Press.

Komorita, S. S., \& Parks, C. D. (1994). Social dilemmas. Dubuque, IA: Brown \& Benchmark.

Koper, G., van Knippenberg, D., Bouhuijs, F., Vermunt, R., \& Wilke, H. (1993). Procedural fairness and self-esteem. European Journal of Social Psychology, 23, 313-325.

Kramer, R. M. (1991). Intergroup relations and organizational dilemmas: The role of categorization processes. In B. M. Staw \& L. L. Cummings (Eds.), Research in Organizational Behavior (Vol. 13, pp. 191-228). Greenwich, CT: JAI Press.

Leventhal, G. S. (1980). What should be done with equity theory? In K. Gergen, M. Greenberg, \& R. Willis (Eds.), Social exchange theory (pp. 27-55). New York: Plenum.

Levine, T. R., Breshnahan, M. J., Park, H. S., Lapinski, M. K., Wittenbaum, G. M., Shearman, S. M., Lee, S. Y., Chung, D., \& Ohashi, R.
(2003). Self-construal scales lack validity. Human Communication Research, 29, 210-252.

Lind, E. A., Kray, L., \& Thompson, L. (1998). The social construction of injustice: Fairness judgments in response to own and others' unfair treatment by authorities. Organizational Behavior and Human Decision Processes, 75, 1-22.

Lind, E. A., \& Tyler, T. R. (1988). The social psychology of procedural justice. New York: Plenum Press.

Markus, H., \& Kitayama, S. (1991). Culture and the self: Implications for cognition, emotion, and motivation. Psychological Review, 98, 224-253.

McFarlin, D. B., \& Sweeney, P. D. (1992). Distributive and procedural justice as predictors of satisfaction with personal and organizational outcomes. Academy of Management Journal, 35, 626-637.

Mikula, G., Scherer, K. R., \& Athenstaedt, U. (1998). The role of injustice in the elicitation of differential emotional reactions. Personality and Social Psychology Bulletin, 24, 769-783.

Moorman, R. H. (1991). Relationship between organizational justice and organizational citizenship behaviors: Do fairness perceptions influence employee citizenship?. Journal of Applied Psychology, 76, 845-855.

Oyserman, D., Coon, H. M., \& Kemmelmeier, M. (2002). Rethinking individualism and collectivism: Evaluation of theoretical assumptions and meta-analyses. Psychological Bulletin, 128, 3-72.

Rosenberg, M. (1965). Society and the adolescent self-image. Princeton, NJ: Princeton University Press.

Singelis, T. M. (1994). The measurement of independent and interdependent self-construal. Personality and Social Psychology Bulletin, 20, 580-591.

Smith, K. G., Carroll, S. J., \& Ashford, S. J. (1995). Intra- and interorganizational cooperation: Toward a research agenda. Academy of Management Journal, 38, 7-23.

Thibaut, J., \& Walker, L. (1975). Procedural justice: A psychological analysis. Hillsdale, NJ: Erlbaum.

Tyler, T. R., \& Degoey, P. (1995). Collective restraint in social dilemmas: Procedural justice and social identification effects on support of authorities. Journal of Personality and Social Psychology, 69, 482-497.

Tyler, T. R., \& Smith, H. J. (1998). Social justice and social movements. In D. Gilbert, S. T. Fiske, \& G. Lindzey (Eds.), Handbook of social psychology (Vol. 4, pp. 595-629). Boston, MA: McGraw-Hill.

Van den Bos, K. (1999). What are we talking about when we talk about no-voice procedures? On the psychology of the fair outcome effect. Journal of Experimental Social Psychology, 35, 560-577.

Van den Bos, K. (in press). What is responsible for the fair process effect? In J. Greenberg \& J. A. Colquitt (Eds.), Handbook of organizational justice: Fundamental questions about fairness in the workplace. Mahwah, NJ: Erlbaum.

Van den Bos, K., \& Lind, E. A. (2002). Uncertainty management by means of fairness judgments. In M. P. Zanna (Ed.), Advances in experimental social psychology (Vol. 34, pp. 1-60). San Diego, CA: Academic Press.

Van den Bos, K., \& Miedema, J. (2000). Toward understanding why fairness matters: The influence of mortality salience on reactions to procedural fairness. Journal of Personality and Social Psychology, 79, 355-366.

Van den Bos, K., \& Spruijt, N. (2002). Appropriateness of decisions as a moderator of the psychology of voice. European Journal of Social Psychology, 32, 57-72.

Van den Bos, K., Wilke, H. A. M., \& Lind, E. A. (1998). When do we need procedural fairness? The role of trust in authority. Journal of Personality and Social Psychology, 75, 1449-1458.

Vandello, J. A., \& Cohen, D. (1999). Patterns of individualism and collectivism across the United States. Journal of Personality and Social Psychology, 77, 279-292.

Weiss, H. M., Suckow, K., \& Cropanzano, R. (1999). Effects of justice conditions on discrete emotions. Journal of Applied Psychology, 84, 786-794. 